\title{
A Sufficient Condition for Asymptotic Stability of Discrete-Time Interval System with Delay
}

\author{
Wei Zhu \\ Institute of Applied Mathematics, Chongqing University of Posts and Telecommunications,
} Chongqing 400065, China

Correspondence should be addressed to Wei Zhu, zhu_wei76@126.com

Received 4 September 2007; Revised 24 November 2007; Accepted 7 January 2008

Recommended by Manuel De La Sen

The asymptotic stability of discrete-time interval system with delay is discussed. A new sufficient condition for preserving the asymptotic stability of the system is presented by means of the inequality techniques. By mathematical analysis, the stability criterion is less conservative than that in previous result. Finally, one example is given to demonstrate the applicability of the present scheme.

Copyright (C) 2008 Wei Zhu. This is an open access article distributed under the Creative Commons Attribution License, which permits unrestricted use, distribution, and reproduction in any medium, provided the original work is properly cited.

\section{Introduction}

The stability analysis of interval system is very useful for the robustness analysis of nominally stable system subject to model perturbations. Therefore, there has been considerable interest in the stability analysis of interval system in literature ([1-15], and references therein). In general, those approaches can be classified into two categories: the first is the polynomial and the second is the matrix approach. However, due to information transmission between elements or systems, data computation, natural property of system elements, and so forth, time delays also inherently exist in controlled systems and therefore must be integrated into system models. The stability analysis for interval systems with delays becomes more complicated. In [6], a sufficient condition for the stability of discrete-time systems is given in terms of pulse-response sequence matrix. In [11], based on the Gersgorin theorem, the stability testing problem for continuous and discrete systems including a time delay is discussed.

The objective of this paper is to deal with the asymptotic stability of a discrete-time interval system with delay. Based on the inequality techniques [16], a new sufficient condition for preserving the asymptotic stability of the system is presented. By mathematical analysis, the stability criterion is less conservative than that in previous result. An example is given to compare the proposed method with one reported. 


\section{System description and notations}

Consider the discrete-time interval system with delay described by

$$
\begin{gathered}
x(k+1)=A_{I} x(k)+B_{I} x(k-p), \quad k=0,1,2, \ldots, \\
x(k)=\varphi(k), \quad k=-p, \ldots, 0,
\end{gathered}
$$

where delay $p$ is a positive integer, $x(k)=\operatorname{col}\left\{x_{1}(k), x_{2}(k), \ldots x_{n}(k)\right\} \in R^{n}, A_{I}, B_{I}$ are the interval matrices described as $A_{I} \triangleq[\underline{A}, \bar{A}]=\left\{A=\left(a_{i j}\right): a_{i j} \in\left[\underline{a}_{i j}, \bar{a}_{i j}\right]\right\}, B_{I} \triangleq[\underline{B}, \bar{B}]=\{B=$ $\left.\left(b_{i j}\right): b_{i j} \in\left[\underline{b}_{i j}, \bar{b}_{i j}\right]\right\}, i, j=1,2, \ldots, n, \varphi(k), k=-p, \ldots, 0$, are bounded.

In the sequel, the following notations will be used: $R^{n}\left(R_{+}^{n}\right)$ : the space of $n$-dimensional (nonnegative) real column vectors; $R^{n \times m}\left(R_{+}^{n \times m}\right)$ : the set of $n \times m$ (nonnegative) real matrices; $\rho(A)$ : the spectral radius of matrix $A \in R^{n \times n} ; A \geq B(A>B)$ : each pair of corresponding elements of $A$ and $B$ satisfies the inequality " $\geq(>)$," where $A, B \in R^{n \times m}$ or $A, B \in R^{n} ; W_{\rho}(A)$ : for $A \in R_{+}^{n \times n}, W_{\rho}(A) \triangleq\left\{z \in R_{+}^{n} \mid A z=\rho(A) z\right\} ;[\cdot]^{+}:$the vector (or matrix) obtained by replacing each entry of [.] by its absolute value; $Z^{[a, b]}: Z^{[a, b]} \equiv\{a, a+1, \ldots, b\}$, where $a, b$ are nonnegative integers; if $a>b$, we define $Z^{[a, b]}=\varnothing$, where $\varnothing$ is the empty set; if $b=\infty$, we write $Z^{[a, b]}$ as $Z^{[a]} ; \mathcal{A}_{r}\left(B_{r}\right)$ : the set of matrices obtained by exchanging corresponding $r$ column $(s)$ of $\underline{A}(\underline{B})$ and $\bar{A}(\bar{B})$, so there are $2 \times\left(\begin{array}{c}n \\ r\end{array}\right)$ matrices in each $\mathcal{A}_{r}\left(\mathbb{B}_{r}\right)$, where $r \in Z^{[0, n]}$, $\left(\begin{array}{c}n \\ r\end{array}\right)=n ! / r !(n-r) !$ and $n !$ denotes the factorial of $n$.

\section{Main result}

In order to prove our main result, we first need the following technical lemmas.

Lemma 3.1 (see [17, Theorem 8.3.1]). If $A \in R_{+}^{n \times n}$, then there is a nonnegative vector $z \geq 0, z \neq 0$, such that $A z=\rho(A) z$.

So it is clear that $W_{\rho}(A)$ is not empty by Lemma 3.1.

Lemma 3.2. Let $P, Q \in R_{+}^{n \times n}, u(k) \in R_{+}^{n}$ satisfy that

$$
u(k+1) \leq P u(k)+Q u(k-p), \quad k \in Z^{[0]} .
$$

If

$$
\rho(P+Q)<1
$$

then there exists a constant $\lambda>0$ such that

$$
u(k) \leq z e^{-\lambda k}, \quad k \in Z^{[0]},
$$

for some $z=\left(z_{1}, z_{2}, \ldots, z_{n}\right)^{T} \in W_{\rho}\left(P+Q e^{\lambda p}\right)$.

Proof. Since $\rho(P+Q)<1$, using continuity, there must be a sufficiently small constant $\lambda>0$ such that

$$
e^{\lambda(p+1)} \rho\left(P+Q e^{\lambda p}\right) \leq 1
$$


Let

$$
\begin{aligned}
& y(k)=u(k) e^{\lambda k}, \quad k \in Z^{[0]}, \\
& y(k)=u(k), \quad k \in Z^{[-p, 0]},
\end{aligned}
$$

so we have

$$
u(k)=y(k) e^{-\lambda k} \leq y(k) e^{-\lambda(k-p)}, \quad k \in Z^{[0]} .
$$

By (3.1), we have

$$
y(k+1)=u(k+1) e^{\lambda(k+1)} \leq(P u(k)+Q u(k-p)) e^{\lambda(k+1)}, \quad k \in Z^{[0]} .
$$

Since $P, Q \in R_{+}^{n \times n}$, we derive that

$$
y(k+1) \leq\left(P y(k)+Q y(k-p) e^{\lambda p}\right) e^{\lambda(p+1)}, \quad k \in Z^{[0]} .
$$

We next show that for any $k \in Z^{[0]}$,

$$
y(k) \leq z
$$

If this is not true, then there must be a positive constant $l>0$ and some integer $m$ such that

$$
y_{m}(l+1)>z_{m}, \quad y(k) \leq z \quad \text { for } k \in Z^{[-p, l]} .
$$

By using (3.4) and (3.8), we obtain that

$$
y(l+1) \leq e^{\lambda(p+1)}\left(P+Q e^{\lambda p}\right) z=e^{\lambda(p+1)} \rho\left(P+Q e^{\lambda p}\right) z \leq z
$$

which contradicts the first inequality of (3.10). Thus (3.9) holds for all $k \in Z^{[0]}$. Therefore, we have

$$
u(k) \leq z e^{-\lambda k}, \quad k \in Z^{[0]},
$$

and the proof is completed.

Theorem 3.3. For any $C \in \mathcal{A} \triangleq \bigcup_{r=0}^{n} \mathcal{A}_{r}, D \in \mathbb{B} \triangleq \bigcup_{r=0}^{n} \mathcal{B}_{r}$, if the inequality

$$
\rho\left([C]^{+}+[D]^{+}\right)<1
$$

holds, then the discrete-time interval system (2.1) is asymptotically stable.

Proof. Let $\Gamma_{k}=\left\{i \mid x_{i}(k)<0\right\} \equiv\left\{i_{1}, i_{2}, \ldots, i_{m}\right\}, \Lambda_{k}=\left\{j \mid x_{j}(k-p)<0\right\} \equiv\left\{j_{1}, j_{2}, \ldots, j_{l}\right\}$, where $m, l$ satisfying $0 \leq m \leq n, 0 \leq l \leq n, m+l=n$ are integers and $m=0$ or $l=0$ is equivalent to that $\Gamma_{k}$ or $\Lambda_{k}$ is empty, respectively. Obviously, $\bigcup_{k=1}^{\infty} \Gamma_{k}$ and $\bigcup_{k=1}^{\infty} \Lambda_{k}$ are finite sets.

By the definitions of $\mathcal{A}$ and $B$, we can obtain matrices $A_{1 k}, A_{2 k} \in \mathcal{A}$ by exchanging the corresponding $i_{1}$ th, $i_{2}$ th $, \ldots, i_{m}$ th columns of $\underline{A}$ and $\bar{A}$ (if $m=0$, then $A_{1 k}=\underline{A}, A_{2 k}=\bar{A}$ ), and 
matrices $B_{1 k}, B_{2 k} \in B$ by exchanging the corresponding $j_{1}$ th, $j_{2}$ th, $\ldots, j_{l}$ th columns of $\underline{B}$ and $\bar{B}$ (if $l=0$, then $B_{1 k}=\underline{B}, B_{2 k}=\bar{B}$ ) such that the following inequalities hold:

$$
\begin{gathered}
A_{1 k} x(k) \leq A x(k) \leq A_{2 k} x(k), \quad A \in A_{I}, \\
B_{1 k} x(k-p) \leq B x(k-p) \leq B_{2 k} x(k-p), \quad B \in B_{I} .
\end{gathered}
$$

So together with (2.1), we have

$$
A_{1 k} x(k)+B_{1 k} x(k-p) \leq x(k+1) \leq A_{2 k} x(k)+B_{2 k} x(k-p) .
$$

From the above, we see that $A_{1 k}, A_{2 k}$ and $B_{1 k}, B_{2 k}$ depend only on the position of the negative components of $x(k)$ and $x(k-p)$, respectively.

Then, from (3.15), we derive

$$
\begin{aligned}
{[x(k+1)]^{+} } & \leq \max \left\{\left[A_{1 k} x(k)+B_{1 k} x(k-p)\right]^{+},\left[A_{2 k} x(k)+B_{2 k} x(k-p)\right]^{+}\right\} \\
& \leq \max \left\{\left[A_{1 k}\right]^{+}[x(k)]^{+}+\left[B_{1 k}\right]^{+}[x(k-p)]^{+},\left[A_{2 k}\right]^{+}[x(k)]^{+}+\left[B_{2 k}\right]^{+}[x(k-p)]^{+}\right\} .
\end{aligned}
$$

So we have

$$
[x(k+1)]^{+} \leq\left[A_{1 k}\right]^{+}[x(k)]^{+}+\left[B_{1 k}\right]^{+}[x(k-p)]^{+}
$$

or

$$
[x(k+1)]^{+} \leq\left[A_{2 k}\right]^{+}[x(k)]^{+}+\left[B_{2 k}\right]^{+}[x(k-p)]^{+} .
$$

Since $A_{1 k}, A_{2 k} \in \mathcal{A}, B_{1 k}, B_{2 k} \in \mathbb{B}$, by the definitions of $\mathcal{A}$ and $B$ again, (3.17) and (3.18), for any $k \in Z^{[0]}$, we can find corresponding matrices $A_{i} \in \mathcal{A}, B_{j} \in B, i, j \in\{1,2, \ldots, n\}$, such that

$$
[x(k+1)]^{+} \leq\left[A_{i}\right]^{+}[x(k)]^{+}+\left[B_{j}\right]^{+}[x(k-p)]^{+} .
$$

In view of condition (3.13), we obtain that

$$
\rho\left(\left[A_{i}\right]^{+}+\left[B_{j}\right]^{+}\right)<1, \quad i, j \in\{1,2, \ldots, n\} .
$$

Thus, by Lemma 3.2 and (3.19), (3.20), for any $k \in Z^{[0]}$, there exist constants $\lambda_{i j}>0$ and some $z_{i j} \in W_{\rho}\left(\left[A_{i}\right]^{+}+\left[B_{j}\right]^{+} e^{\lambda_{i j} p}\right), i, j \in\{1,2, \ldots, n\}$, such that

$$
x(k) \leq z_{i j} e^{-\lambda_{i j} k} .
$$

Set $\lambda=\min _{1 \leq i, j \leq n}\left\{\lambda_{i j}\right\}, z_{i j}=\left\{z_{i j}^{(1)}, z_{i j}^{(2)}, \ldots, z_{i j}^{(n)}\right\}^{T}, z^{(h)}=\max _{1 \leq i, j \leq n}\left\{z_{i j}^{(h)}\right\}, h=1,2, \ldots, n, z=$ $\left\{z^{(1)}, z^{(2)}, \ldots, z^{(n)}\right\}^{T}$, obviously, $\lambda$ and $z$ are independent of any choice of $k$, so by (3.21), we derive that

$$
x(k) \leq z e^{-\lambda k}, \quad k \in Z^{[0]},
$$

which implies that the conclusion of the theorem holds. 
Wei Zhu

Remark 3.4. By the meanings of $\mathcal{A}_{r}$ and $\boldsymbol{B}_{r}$, we know that $\mathcal{A}_{r}=\mathcal{A}_{n-r}, \boldsymbol{B}_{r}=\boldsymbol{B}_{n-r}, r \in Z^{[0, n]}$. So there are $\left(\sum_{r=0}^{n} 2 \times\left(\begin{array}{c}n \\ r\end{array}\right)\right) / 2=2^{n}$ matrices in the sets $\mathcal{A}$ and $B$, respectively. Furthermore, if number $n$ is even, that is, $n=2 k, k=1,2, \ldots$, then the equality $A_{r}=A_{n-r}\left(B_{r}=B_{n-r}\right)$ for $r=k$ is transformed to be $A_{k}=A_{k}\left(B_{k}=B_{k}\right)$ and then $\mathcal{A}_{k}\left(\boldsymbol{B}_{k}\right)$ contains only $\left(\begin{array}{c}2 k \\ k\end{array}\right)$ different matrices. Therefore, condition (3.13) can be verified easily and quickly by computer software (such as MATLAB).

Corollary 3.5 (see [11, Theorem IV]). The discrete-time interval system (2.1) is asymptotically stable if the following condition is satisfied:

$$
\rho(K+F)<1,
$$

where matrices $K$ and $F$ are defined as $K=\left(k_{i j}\right), k_{i j}=\max \left\{\left|\underline{a}_{i j}\right|,\left|\bar{a}_{i j}\right|\right\}, F=\left(f_{i j}\right), f_{i j}=$ $\max \left\{\left|\underline{b}_{i j}\right|,\left|\bar{b}_{i j}\right|\right\}, i, j=1,2, \ldots, n$.

Proof. Clearly, for any $C \in \mathscr{A}=\bigcup_{i=1}^{n} \mathcal{A}_{i}, D \in \mathbb{B}=\bigcup_{i=1}^{n} \boldsymbol{B}_{i}$, the inequality

$$
[C]^{+}+[D]^{+} \leq K+F
$$

holds, then from [18] (i.e., for $A, B \in R_{+}^{n \times n}$, if $A \leq B$, then $\rho(A) \leq \rho(B)$ ) and associated with (3.23), we have

$$
\rho\left([C]^{+}+[D]^{+}\right) \leq \rho(K+F)<1 .
$$

Therefore, system (2.1) is asymptotically stable in terms of Theorem 3.3.

\section{Illustrative examples}

Example 4.1. Consider the discrete-time interval system (2.1) with delay and

$$
\underline{A}=\left(\begin{array}{cc}
-\frac{1}{2} & -\frac{1}{4} \\
0 & \frac{1}{4}
\end{array}\right), \quad \bar{A}=\left(\begin{array}{cc}
-\frac{1}{3} & 0 \\
\frac{1}{4} & \frac{1}{2}
\end{array}\right), \quad \underline{B}=\left(\begin{array}{cc}
-\frac{1}{4} & -\frac{1}{6} \\
-\frac{1}{4} & -\frac{1}{8}
\end{array}\right), \quad \bar{B}=\left(\begin{array}{cc}
-\frac{1}{5} & 0 \\
0 & \frac{1}{10}
\end{array}\right) .
$$

For this case, $\mathcal{A}_{1}=\left\{A_{1}=\left(\begin{array}{cc}-1 / 3 & -1 / 4 \\ 1 / 4 & 1 / 4\end{array}\right), A_{2}=\left(\begin{array}{cc}-1 / 2 & 0 \\ 0 & 1 / 2\end{array}\right)\right\}$,

$$
\begin{aligned}
& A_{2}=\left\{A_{3}=\left(\begin{array}{cc}
-\frac{1}{3} & 0 \\
\frac{1}{4} & \frac{1}{2}
\end{array}\right), A_{4}=\left(\begin{array}{cc}
-\frac{1}{2} & -\frac{1}{4} \\
0 & \frac{1}{4}
\end{array}\right)\right\}, \\
& B_{1}=\left\{B_{1}=\left(\begin{array}{rr}
-\frac{1}{5} & -\frac{1}{6} \\
0 & -\frac{1}{8}
\end{array}\right), B_{2}=\left(\begin{array}{rr}
-\frac{1}{4} & 0 \\
-\frac{1}{4} & \frac{1}{10}
\end{array}\right)\right\}, \\
& B_{2}=\left\{B_{3}=\left(\begin{array}{rr}
-\frac{1}{5} & 0 \\
0 & \frac{1}{10}
\end{array}\right), B_{4}=\left(\begin{array}{rr}
-\frac{1}{4} & -\frac{1}{6} \\
-\frac{1}{4} & -\frac{1}{8}
\end{array}\right)\right\} .
\end{aligned}
$$




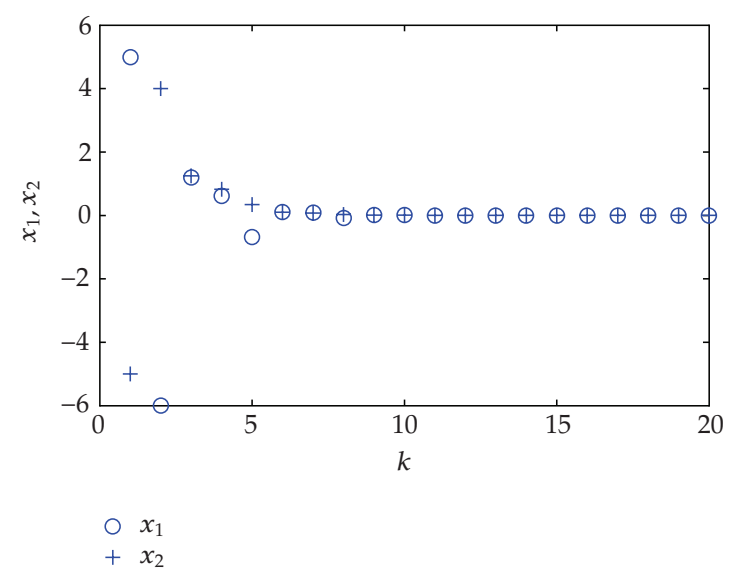

Figure 1: Stability for discrete-time interval system.

By simple calculation, we have $\rho\left(\left[A_{i}\right]^{+}+\left[B_{j}\right]^{+}\right) \leq \rho\left(\left[A_{1}\right]^{+}+\left[B_{4}\right]^{+}\right)$for $i, j=1,2,3,4$, and

$$
\left[A_{1}\right]^{+}+\left[B_{4}\right]^{+}=\left(\begin{array}{cc}
\frac{1}{3} & \frac{1}{4} \\
\frac{1}{4} & \frac{1}{4}
\end{array}\right)+\left(\begin{array}{cc}
\frac{1}{4} & \frac{1}{6} \\
\frac{1}{4} & \frac{1}{8}
\end{array}\right)=\left(\begin{array}{cc}
\frac{7}{12} & \frac{5}{12} \\
\frac{1}{2} & \frac{3}{8}
\end{array}\right) .
$$

So we have

$$
\rho\left(\left[A_{i}\right]^{+}+\left[B_{j}\right]^{+}\right) \leq \rho\left(\left[A_{1}\right]^{+}+\left[B_{4}\right]^{+}\right)=0.9473<1, \quad i, j=1,2,3,4 .
$$

Therefore, the system (2.1) is asymptotically stable by means of Theorem 3.3.

In what follows, the simulation result is illustrated in Figure 1.

Remark 4.2. If [11, Theorem IV] is applied to Example 4.1, we obtain

$$
K=\left(\begin{array}{cc}
\frac{1}{2} & \frac{1}{4} \\
\frac{1}{4} & \frac{1}{2}
\end{array}\right), \quad F=\left(\begin{array}{cc}
\frac{1}{4} & \frac{1}{6} \\
\frac{1}{4} & \frac{1}{8}
\end{array}\right)
$$

where $K, F$ are defined by Corollary 3.5, that is, [11, Theorem IV]. Then

$$
\rho(K+F)=1.1482>1,
$$

that is, [11, Theorem IV] cannot be applied. So the sufficient condition (3.13) proposed in this paper is less conservative than condition (3.23) proposed by [11].

\section{Conclusion}

In this paper, we have investigated the asymptotic stability of discrete-time interval system with delay. A new sufficient condition for preserving the asymptotic stability of the system is 
developed. By mathematical analysis, the presented criterion is to be less conservative than that proposed by [11]. So, the result of this paper indeed allows us to have more freedom for checking the stability of the discrete-time interval systems with delay. From the proposed example, it is easily seen that the criterion presented in this paper for the stability of the discrete-time interval system with delay is very helpful. We believe that the present scheme is applicable to robust control design.

\section{Acknowledgments}

The author wishes to thank the editor and the referees for their helpful and interesting comments. The work is supported by National Natural Science Foundation of China under Grant 10671133 and the Doctor's Foundation of Chongqing University of Posts and Telecommunications A2007-41.

\section{References}

[1] S. Białas, "A necessary and sufficient condition for the stability of interval matrices," International Journal of Control, vol. 37, no. 4, pp. 717-722, 1983.

[2] R. K. Yedavalli, "Stability analysis of interval matrices: another sufficient condition," International Journal of Control, vol. 43, no. 3, pp. 767-772, 1986.

[3] J. Chen, "Sufficient conditions on stability of interval matrices: connections and new results," IEEE Transactions on Automatic Control, vol. 37, no. 4, pp. 541-544, 1992.

[4] D. Y. Xu, "Simple criteria for stability of interval matrices," International Journal of Control, vol. 41, no. 1, pp. 289-295, 1985.

[5] M.-H. Shih, Y.-Y. Lur, and C.-T. Pang, "An inequality for the spectral radius of an interval matrix," Linear Algebra and Its Applications, vol. 274, pp. 27-36, 1998.

[6] P.-L. Liu and W.-J. Shyr, "Another sufficient condition for the stability of grey discrete-time systems," Journal of the Franklin Institute, vol. 342, no. 1, pp. 15-23, 2005.

[7] K. Yamac and D. Bozkurt, "On stability of discrete-time interval matrices," Applied Mathematics and Computation, vol. 191, pp. 299-301, 2007.

[8] C. L. Jiang, "Sufficient condition for the asymptotic stability of interval matrices," International Journal of Control, vol. 46, no. 5, pp. 1803-1810, 1987.

[9] J. L. Deng, “Introduction to grey system theory,” Journal of Grey System, vol. 1, no. 1, pp. 1-24, 1989.

[10] C. S. Zhou, "Sufficient criteria for stability analysis of grey discrete-time systems," Journal of Grey System, vol. 7, no. 1, pp. 45-50, 1995.

[11] C.-H. Lee and T.-L. Hsien, "New sufficient conditions for the stability of continuous and discrete timedelay interval systems," Journal of the Franklin Institute, vol. 334, no. 2, pp. 233-240, 1997.

[12] Y.-T. Juang, T.-S. Kuo, and S.-L. Tung, "Stability analysis of continuous and discrete interval systems," Control Theory and Advanced Technology, vol. 6, no. 2, pp. 221-235, 1990.

[13] M. De La Sen, "On the properties of reachability, observability, controllability, and constructibility of discrete-time positive time-invariant linear systems with aperiodic choice of the sampling instants," Discrete Dynamics in Nature and Society, vol. 2007, Article ID 84913, 23 pages, 2007.

[14] M. De La Sen, "Stability and assignment of spectrum in systems with discrete time lags," Discrete Dynamics in Nature and Society, vol. 2006, Article ID 76361, 8 pages, 2006.

[15] L. Shaikhet, "A new view on one problem of asymptotic behavior of solutions of delay difference equations," Discrete Dynamics in Nature and Society, vol. 2006, Article ID 74043, 16 pages, 2006.

[16] D. Y. Xu, Z. X. Ma, and Q. Y. Guo, "Difference inequalities and domains of attraction of discrete systems," Chinese Annals of Mathematics. Series A, vol. 20, no. 1, pp. 21-26, 1999 (Chinese).

[17] R. A. Horn and C. R. Johnson, Matrix Analysis, Cambridge University Press, Cambridge, UK, 1985.

[18] J. M. Ortega, Numerical Analysis. A Second Course, Computer Science and Applied Mathematics, Academic Press, New York, NY, USA, 1972. 


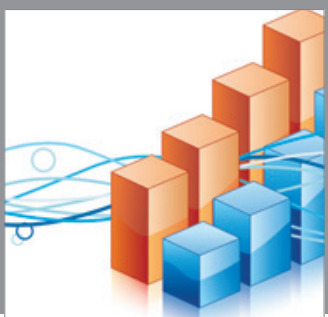

Advances in

Operations Research

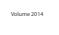

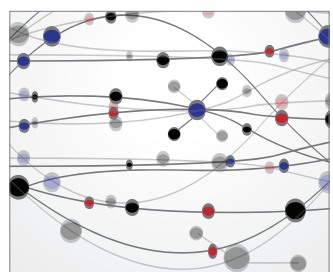

\section{The Scientific} World Journal
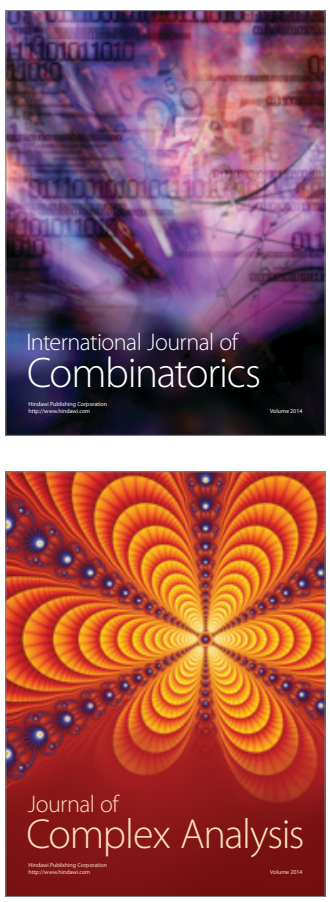

International Journal of

Mathematics and

Mathematical

Sciences
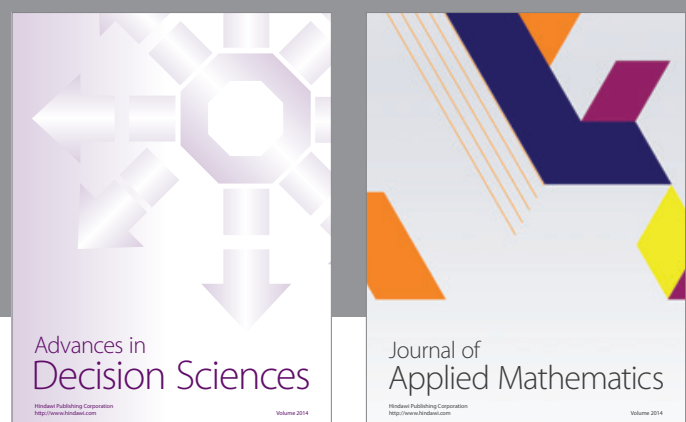

Journal of

Applied Mathematics
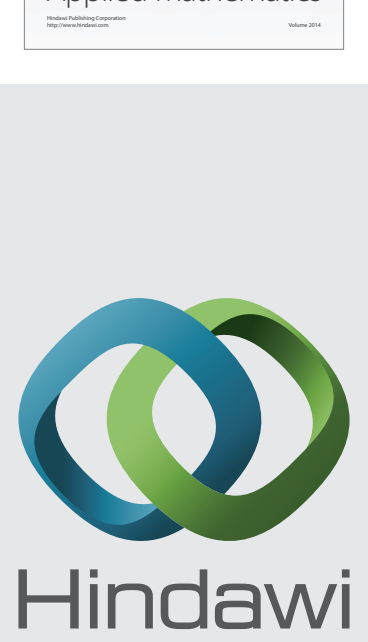

Submit your manuscripts at http://www.hindawi.com
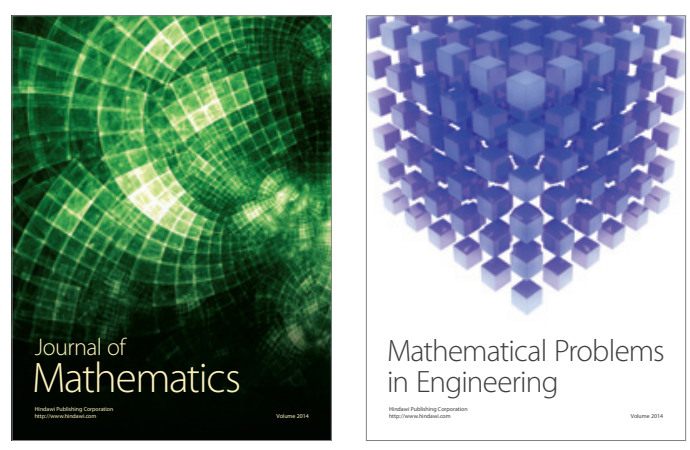

Mathematical Problems in Engineering
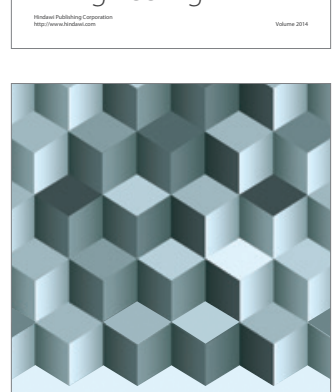

Journal of

Function Spaces
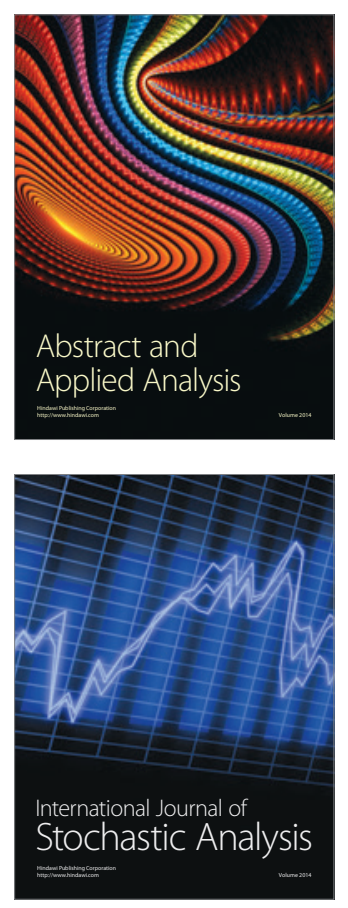

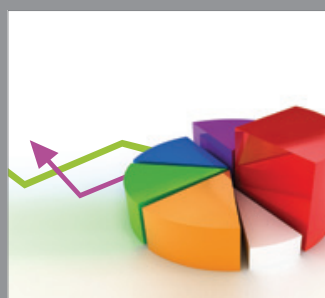

ournal of

Probability and Statistics

Promensencen
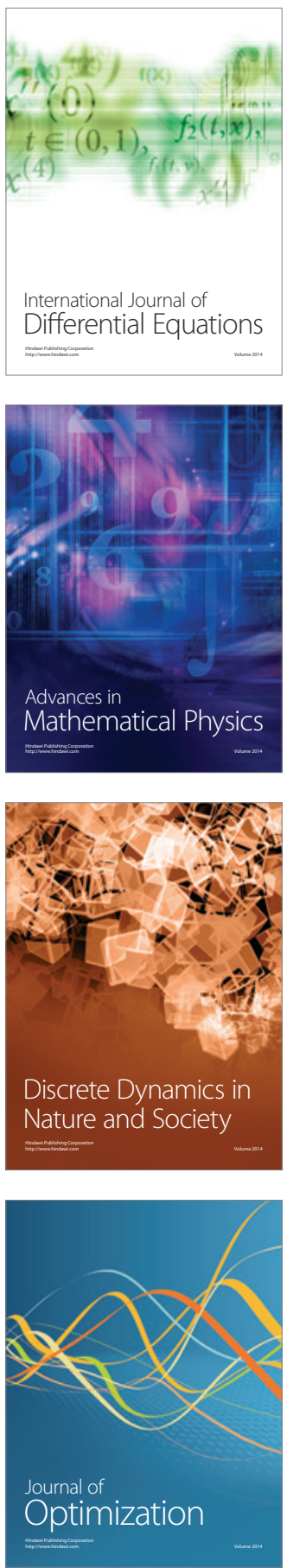